\title{
Confort visual en oficinas, factor temporal en la evaluación de deslumbramiento
}

\section{Visual comfort in office work, temporal factor in Glare evaluation}

\author{
$\underline{\text { J. Yamin }}^{(*)}$, A. Pattini ${ }^{(* *)}$, E. Colombo ${ }^{(* * *)}$
}

\section{RESUMEN}

Para lograr los beneficios de iluminar con luz natural espacios de trabajo, hay que evitar que la luz natural sea bloqueada por el potencial riesgo de deslumbramiento. Para lograr una adecuada caracterización del mismo es importante considerar la percepción del tiempo de exposición de la fuente en el campo de visión (Aspecto no incluido en los modelos de deslumbramiento). Para determinar de qué manera influye este aspecto perceptual en la sensación de deslumbramiento se evaluaron dos escenarios con dos tipos de manchas solares sobre el puesto de trabajo: 1) Con mayor profundidad de ingreso y más tiempo en el campo de visión, 2) Con menor profundidad de ingreso y menos tiempo en el campo de visión. Se midieron parámetros subjetivos (GSV) y variables fotométricas (DGP, EV y CL). Siendo la principal variable explicativa la EV (rho=0.51). Observándose también una mayor tolerancia al deslumbramiento por el efecto transitorio de la fuente.

Palabras clave: Iluminación natural, deslumbramiento, trabajo de oficinas, confort visual.

\section{ABSTRACT}

In order to achieve the benefits of daylight to illuminate work spaces, the daylight blocking should be avoided by the potential risk of glare. To achieve an adequate characterization of glare, it is important to consider the perception of the exposure time of the source in the field of vision (Aspect not included in the glare models). In order to determine how this perceptual aspect influences the glare sensation, two scenarios with two types of sunspots on the workplace were evaluated: 1) with greater depth of direct light income and more time in the field of view, 2) with less depth of entry and less time in the field of view. Behavioral parameters, subjective surveys (GSV) and photometric variables (DGP, EV and LR) were measured. The EV was the main explanatory variable (rho $=0.51)$. Also it was observed a greater tolerance to glare due to the transitory effect of the source.

Keywords: Daylight, glare, office work, visual comfort.

(*) Dr. Medio Ambiente Visual e Iluminación eficiente. Investigador Asistente. INAHE-Conicet, Mendoza (Argentina)

${ }^{(* *)}$ Dr. Medio Ambiente Visual e Iluminación eficiente. Investigador Principal. INAHE-Conicet, Mendoza (Argentina)

(***) Dr. Medio Ambiente Visual e Iluminación eficiente. Investigador Principal. ILAV-FACET-Conicet, Tucumán (Argentina)

Persona de contacto / Corresponding author: jyamin@mendoza-conicet.gob.ar (J. Yamin)

ORCID: https://orcid.org/oooo-0oo1-9322-2902 (J. Yamin); https://orcid.org/oooo-0001-6305-1268 (A. Pattini); https:// orcid.org/oooo-0003-0822-4589 (E. Colombo)

Cómo citar este artículo/Citation: Yamin, J.; Pattini, A.; Colombo, E. (2020). Confort visual en oficinas, factor temporal en la evaluación de deslumbramiento. Informes de la Construcción, 72(557): e329. https://doi.org/10.3989/ic.67992

Copyright: (c) 2020 CSIC. Este es un artículo de acceso abierto distribuido bajo los términos de la licencia de uso y distribución Creative Commons Reconocimiento 4.0 Internacional (CC BY 4.0). 


\section{INTRODUCCIÓN}

En las zonas geográficas con clima árido donde predominan los cielos claros como el caso de la provincia de Mendoza ( $\mathrm{Ar}$ gentina), (latitud $32^{\circ} 53^{\prime} \mathrm{S}$; longitud $68^{\circ} 52^{\prime} \mathrm{O}$ ) hay una gran disponibilidad de luz natural. En estas zonas se registran valores de iluminancia horizontal global muy elevados (Eh global directa: 1200oolx; Eh global difusa: 6000olx) (1). Esta enorme disponibilidad de luz natural además de contribuir en importantes ahorros de energía eléctrica, puede mejorar la calidad ambiental interior del espacio construido (2). Para lograr estos beneficios es necesario un adecuado control solar-lumínico, como así también una adecuada interacción entre ambos. Cuando este control solar no es adecuado, las personas bloquean los aportes de iluminación natural debido al potencial riesgo de deslumbramiento utilizando la luz artificial para iluminar, aumentando los consumos de energía eléctrica (3). Para que la luz natural no sea bloqueada, hay que prestar principal atención al deslumbramiento, identificando parámetros y métodos que permitan obtener una adecuada caracterización del mismo.

Los modelos de deslumbramiento psicológico describen la magnitud subjetiva de deslumbramiento experimentado por los observadores. La mayoría de estas ecuaciones derivan de experimentos realizados con luz artificial, y están compuestos de cuatro variables principales: Luminancia de la fuente, tamaño de la fuente, luminancia del fondo, índice de posición (4) e iluminancia vertical a nivel de los ojos (5). El deslumbramiento no depende solamente de valores absolutos, sino también de valores relativos, usualmente expresado como relaciones de luminancias (4). El modelo de deslumbramiento molesto para luz natural más validado internacionalmente en trabajo de oficina es el índice Probabilidad de Deslumbramiento con Luz Natural (DGP) del inglés "Daylight Glare Probability" $(5,6)$. Este modelo no puede explicar adecuadamente la alta variabilidad existente entre las personas debido a que los mecanismos que gobiernan la sensación de deslumbramiento todavía no están bien identificados (7).

A lo largo de los años se han identificado factores que podrían influir en la sensación de deslumbramiento molesto, pero que aún no son introducidos de manera sistemática y unificada en los estudios del mismo, estos son: diferencias culturales (8), tipo de vista al exterior (9), estado de ánimo, nivel cognitivo y preferencias (10) sensibilidad al deslumbramiento (11), apertura ocular (12) influencia de la temperatura interior (13) percepción de los factores temporales (14).

Los factores temporales en el deslumbramiento se definen como la duración de una fuente de deslumbramiento (tiempo de exposición) en el campo visual. Este factor no ha sido contemplado en los modelos de deslumbramiento psicológico (15). Sin embargo, algunos estudios si consideran la influencia del tiempo en la sensación de deslumbramiento, asociándolo a efectos negativos como el incremento de la sensación de deslumbramiento o positivos como la reducción de la sensación de deslumbramiento, definidos a continuación: -Reducción de la sensación de deslumbramiento: Mcfarlane considera que el tiempo tendría un efecto positivo en la percepción de deslumbramiento. Su estudio sostiene que existe una sobreestimación del deslumbramiento en los modelos de Hopkinson (BGI, DGI). Esta sobreestimación es debido a la habituación (16). Con el término habituación, se refiere a la reducción de la respuesta del organismo a una estimulación repetida. Estudios más recientes encontraron un incremento en la tolerancia al deslumbramiento con luz natural en el transcurso del día, es decir que el deslumbramiento era mejor tolerado durante la tarde que durante la mañana $(14,17)$. Incremento de la sensación de deslumbramiento: Osterhaus (1992) observó que los sujetos experimentales se volvieron cada vez más sensibles al deslumbramiento a medida que el experimento avanzaba en el tiempo (1-1/2 horas). Este experimento fue confirmado por el autor con datos experimentales posteriores $(18,19)$. Frente a esta disparidad de resultados surge la necesidad de determinar cuál es el efecto del tiempo de exposición a la fuente cuando esta es de origen natural.

Mientras varios autores sostienen que una fuente transitoria tiene un efecto de incremento en la percepción de deslumbramiento, la hipótesis de este trabajo sostiene que el conocimiento de la duración de una mancha solar en la escena por parte de los usuarios puede ayudar a disminuir la sensación de deslumbramiento. En este trabajo se prevé determinar si la sensación de deslumbramiento experimentado por los participantes es un fenómeno adaptativo (cuando el ojo se adapta y disminuye la sensación de deslumbramiento) o acumulativo (cuando hay un incremento en la molestia a un tiempo de exposición constante) en función del tiempo de exposición de la fuente. Con el objetivo final de determinar si el factor tiempo de exposición es un parámetro que debería incluirse en los modelos de deslumbramiento.

\section{MATERIAL Y MÉTODO}

\subsection{Caso de estudio}

Para este estudio se seleccionaron 7 oficinas con ingreso de luz solar directa en el puesto de trabajo. Las oficinas se encuentran emplazadas en el centro científico tecnológico CCTMendoza, en planta alta orientadas al este. La dimensión de las oficinas es de $4.62 \times 2.32 \mathrm{~m}$, con una abertura de $1.56 \times 1.8 \mathrm{~m}$ compuesta de tres paños (Figura 1). El elemento de control solar común a todas las oficinas son parasoles horizontales exteriores móviles (Figura 2 y 3) estos son de aluminio, color blanco y reflectancia $\mathrm{r}=0.85$. El elemento de control solar que varía en cada oficina son cortinas ubicadas en el paño 1. Las ventanas se encontraban adyacentes a los puestos de trabajo, ubicándose la pantalla de visualización de datos (PVD) de manera lateral. Respecto a la iluminación artificial, estas consistían en tubos fluorescentes con difusor y estuvieron encendidas durante todo el relevamiento.

\subsection{Participantes}

Los participantes fueron 7 sujetos de entre 25 y 30 años de edad $(\mathrm{m}=36$; $\mathrm{Ds}=11)$. Esta muestra de 7 personas no se eligió al azar. Los sujetos experimentales fueron seleccionados de una muestra de mayor tamaño $(n=26)(20)$, pasando por una serie de pruebas estadísticas epidemiológicas de especificidad y sensibilidad. A partir de estas pruebas, fueron clasificados como sujetos experimentados y seleccionados para el experimento. Esta metodología de trabajar con un grupo reducido pero experimentado ha sido validada en algunos trabajos de deslumbramiento $(21,22)$. Cabe mencionar que los 7 sujetos fueron medidos cada media hora en dos períodos estacionales obteniéndose un total de 56 condiciones lumínicas. 


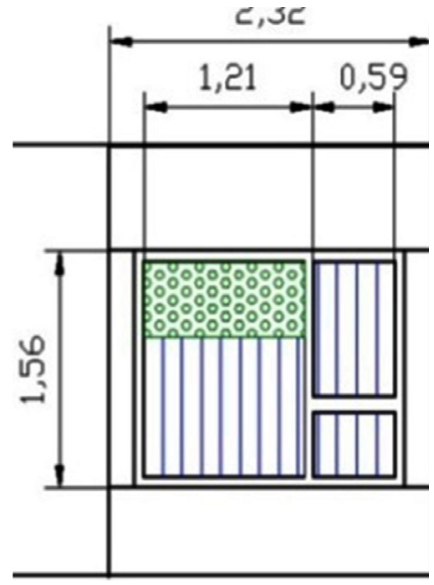

Figura 1. Fachada de las oficinas.

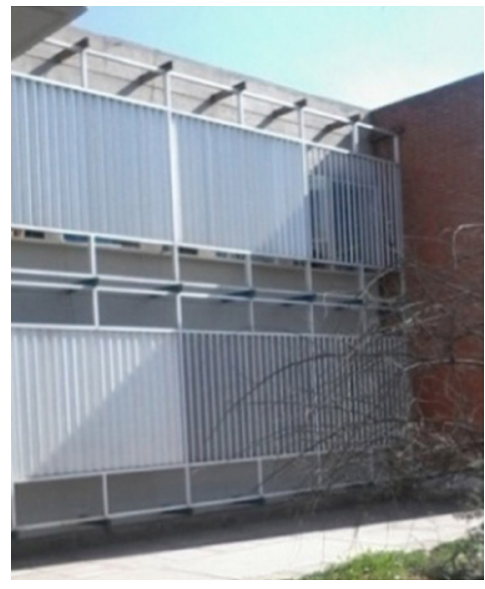

Figura 2. Parasoles exteriores.

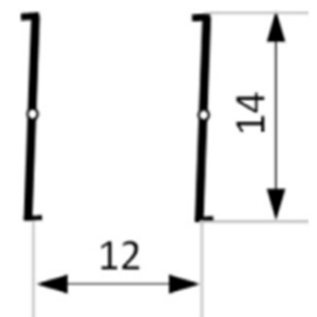

Figura 3. Dimensiones de los parasoles exteriores.
En el experimento, cada participante debía trabajar con su Pantalla de Visualización de Datos (PVD), realizando sus tareas habituales, se le proporcionó un cuestionario y posteriormente se tomaron las medidas fotométricas. Antes de iniciar la toma de datos se les proporcionó a los participantes un formulario consentimiento informado.

\subsection{Casos de estudio. Simulación}

Los meses seleccionados fueron los meses de septiembre y diciembre. Primero se realizaron una serie de simulaciones anuales en el programa Velux Daylight Visualizer 2.o. El objetivo de esta simulación fue conocer la trayectoria del sol en el espacio a evaluar, se utilizaron como "imputs" la geometría del espacio, la latitud: $32^{\circ} 52^{\prime} \mathrm{S}$ y longitud $68^{\circ} 51^{\prime}$. A partir de los datos de la simulación podemos conocer a priori cuáles son los meses en que la mancha de luz difiere en profundidad de ingreso y tiempo de duración. En septiembre la posición de sol es más baja respecto a diciembre, por lo tanto el ingreso de luz solar directa es mayor. Para el mes de septiembre, la altitud y azimut solar produjeron una mancha con mayor profundidad de ingreso, que a su vez estuvo más tiempo sobre la tarea (desde las 9: oo hasta las 11:00 a.m.). Por el contrario, en diciembre, el sol tiene una mayor altitud lo que se traduce en una penetración de la mancha menor, y que a su vez estuvo menos tiempo sobre la tarea (desde las 9:0o hasta las 10:00). Es importante destacar que no hubo variaciones en el uso de la luz eléctrica en las dos condiciones, en ambas estas estuvieron encendidas.

\subsection{Diseño Experimental}

En primer lugar se estudiaron algunos de los factores que según la comunidad científica afectan a la sensación de deslumbramiento (Iluminancias, contraste de luminancias y modelo de deslumbramiento DGP). En segundo lugar se calculó cuánto afecta cada uno de estos factores a la sensación de deslumbramiento. La variación en la sensación de deslumbramiento no explicada por estos factores podría ser atribuida al efecto de temporalidad de la mancha.

La variable a la cual ajustar los datos es la sensación de deslumbramiento percibida (GSV).

\subsection{Medidas Fotométricas}

Iluminancias: Medición al inicio de la tarea de iluminancia vertical a nivel del ojo (Ev) (un punto de medición). Se midió con un luxímetro con sensor de iluminancia marca LMT con rango de 0.1 a 120000 lux con corrector de coseno y filtro $\mathrm{v}$ de lambda.

Uniformidad de Iluminancias: Para calcular la uniformidad de iluminancias, se midió la iluminancia horizontal en el plano de trabajo a partir de tres puntos a distancias regulares y a $0.85 \mathrm{~m}$ del suelo. Esto nos permitió obtener la uniformidad de iluminancias mediante la siguiente fórmula [1]:

$$
\mathrm{Eh}_{\min } / \mathrm{Ehmax}=>0.5<0.7
$$

Dónde: $\mathrm{Eh}_{\min }$ es la iluminancia mínima en el plano de trabajo y $\mathrm{Eh}_{\max }$ es la iluminancia máxima en el plano de trabajo.

Contraste de luminancias (CL) y modelo de DGP: Estas medidas se obtuvieron a partir de imágenes de alto rango dinámico (HDR)(23), tomadas cada 30 minutos para los 7 participantes en las dos situaciones lumínicas evaluadas (septiembre y diciembre). Para este análisis se utilizaron 4 tipos de HDR 1-Escena completa para el cálculo de deslumbramiento (Figura 4a), 2- Escena con campo de visión central (tarea) (Figura 4b), 3- Escena que comprende el campo de visión cercano (Figura 4c) y 4- Escena que comprende el campo de visión lejano (Figura 4d). Obteniéndose un total de 224 HDR.

Modelo DGP: Se calculó a partir del post-procesamiento de las HDR con el programa "Evalglare" (versión 1.21) desarrollado por Wienold (5). El criterio utilizado para detectar la fuente de deslumbramiento fue en función del umbral de luminancia media de la tarea, se utilizó como valor umbral 5, ya que es el método recomendado porque tiene en cuenta la adaptación del observador (5). Una vez detectada la fuente, Evalglare calcula los otros parámetros de la ecuación (luminancia de la ventana, tamaño de la fuente y factor de posición).

Línea de comando utilizada: evalglare -T xpos ypos tamaño -d -c checkfile.hdr input.hdr / evalglare -i valor input.hdr.

Interpretación de comandos (24): (-T) este factor define la posición de la tarea en las coordenadas x,y, y su ángulo de apertura en radianes, (-b) este factor multiplicado por la luminancia promedio en toda la imagen se utilizará como umbral para detectar las fuentes de deslumbramiento, valor por defecto $5,(-c)$ este comando escribe un archivo de veri- 

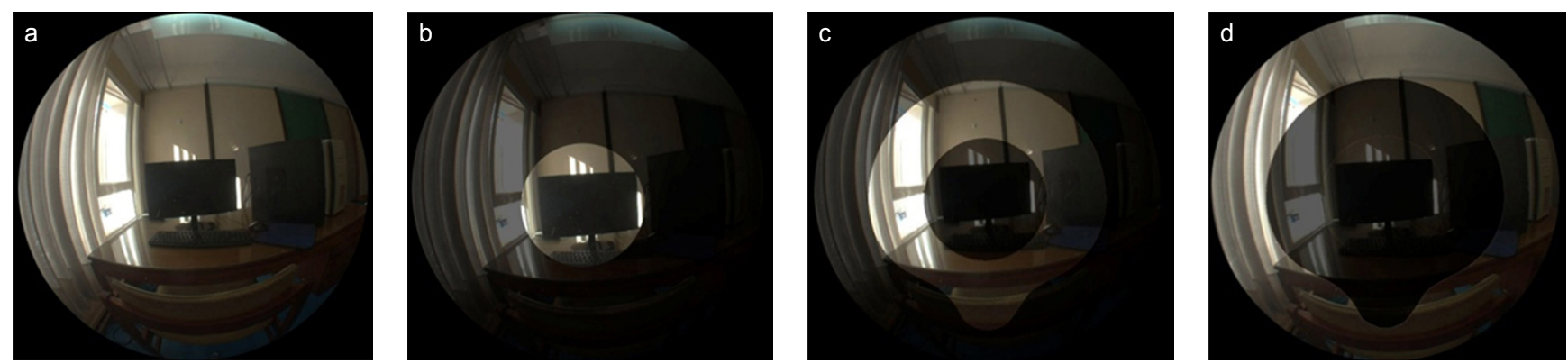

Figura 4. a. Escena completa. b. Campo de visión central (tarea). (Lt). c. Campo de visión cercano. (Ls-cvc).

d. Campo de visión lejano. (Ls-cvl).

ficación en el formato de imagen RADIANCE, (-i) este factor introduce la iluminancia vertical Ev en lux que es medida externamente. Este valor se utilizará para calcular el DGP. Índices de DGP: imperceptible (<0.30), Notable (0.30-0.35) Perturbador (0.35-0.45) Intolerable (>0.45).

Contraste de luminancias: Con el objetivo de calcular los valores de luminancia, se utilizó el programa de código abierto HDRscope (versión 1.0) (25). Las relaciones de luminancias se calcularon mediante el promedio de luminancias de la fuente en relación al promedio de luminancias de la tarea. $\mathrm{El}$ área de la fuente y de la tarea fue determinada mediante máscaras dentro del campo de visión (Figura 4b-c-d). Los valores de referencia son: 1:10 (tarea: campo de visión cercano) y 1:20 (tarea: campo de visión lejano) (26).

\subsection{Registro del uso de parasoles}

Se registró el uso de parasoles cada 30 minutos, para conocer en qué horario los usuarios bloquearon o permitieron el ingreso de sol para los meses de septiembre y diciembre; y en qué posición ajustaron los elementos de control solar (Figura 5).

\subsection{Cuestionario y escala GSV}

La evaluación subjetiva de confort visual se realizó mediante una encuesta que consta de diferenciales semánticos y preguntas de múltiple opción. La encuesta consta de 6 preguntas. En este trabajo se utilizará solamente la pregunta Q1 que mide el nivel de deslumbramiento percibido: (Q1 ¿Qué grado de deslumbramiento experimentó al leer en la pantalla?), el cual se midió con la escala GSV (Glare Sensation Vote) (27). Esta escala fue presentada a los participantes cada media hora mediante una escala ordinal de 4 puntos: 1- imperceptible, 2- notable, 3- perturbador y 4- intolerable. La definición de cada punto de la escala fue presentada a los participantes. Cada categoría de deslumbramiento está asociada al periodo de tiempo aproximado en que el sujeto puede tolerar el deslumbramiento.
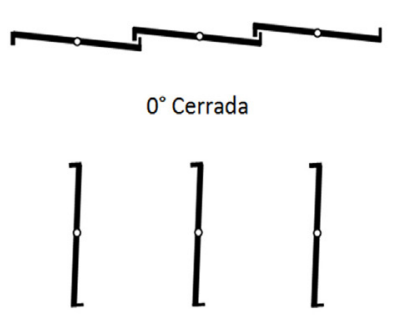

$90^{\circ}$ Abierta

Figura 5. Posición de los parasoles exteriores.

\subsection{Herramientas estadísticas aplicadas sobre los datos}

Se realizó un diseño intra-sujetos ya que se evalúa el comportamiento del mismo grupo de sujetos bajo dos condiciones diferentes. Se emplearon pruebas no paramétricas, ya que estas no requieren conocer la distribución de la muestra y son una alternativa a las pruebas paramétricas cuando los datos no cumplen los requisitos de las pruebas paramétricas (normalidad, homocedasticidad). Estas pruebas también se emplean cuando el tamaño de la muestra es menor a 20 sujetos (28), como en el caso de nuestro estudio. Se aplica generalmente a las variables ordinales o categóricas.

Para medir la relación lineal entre dos variables aleatorias se utilizó el coeficiente de correlación de Spearman (rho). Para medir la porción de la varianza de la variable dependiente explicada por la variable independiente se utilizó el coeficiente pseudo $\mathrm{R}^{2}$ de Nagelkerke $\left(\mathrm{R}^{2}\right)$. La varianza no explicada por las variables explicativas es atribuida al factor temporal.

\section{RESULTADOS}

La tabla 1 muestra la moda de Q1 (GSV), es decir los valores de deslumbramiento percibido que más se repitieron para cada periodo horario en ambos meses.

Respecto a la sensación de deslumbramiento en septiembre, al inicio de la jornada entre las 9:00 y 9:30, el deslumbramiento percibido fue el mayor registrado, "perturbador" y "notable", luego fue disminuyendo, a las 10:00 fue "notable" y a las 10:30 y 11:00 fue "imperceptible". Por el contrario, en diciembre, la sensación de deslumbramiento fue alternando entre "notable" e "imperceptible", sin registrarse casos de deslumbramiento perturbador. A partir de los valores de Q1 (GSV) obtenidos cada media hora se pueden realizar algunas inferencias relacionadas a la hipótesis. Esta variación de la sensación de deslumbramiento a lo largo de la jornada en el mes de septiembre podría indicar que las personas toleraron tener periodos con deslumbramiento al inicio de la jornada.

Efecto que no se vio en el mes de diciembre ya que por motivos térmicos los oficinistas bloquearon el ingreso de radiación directa.

Tabla 1. Moda de la sensación de deslumbramiento.

\begin{tabular}{|l|c|c|c|c|c|}
\cline { 2 - 6 } \multicolumn{1}{c|}{} & GSV & GSV & GSV & GSV & GSV \\
& $9: 00$ & $9: 30$ & $10: 00$ & 10:30 & 11:00 \\
\hline Moda septiembre & $2-3$ & $2-3$ & 2 & 1 & 1 \\
\hline Moda diciembre & 2 & 1 & 2 & 1 & 1 \\
\hline
\end{tabular}


Tabla 2. Valores promedio de iluminancia vertical media para los meses evaluados.

\begin{tabular}{|c|c|c|c|c|c|c|c|c|c|}
\cline { 3 - 10 } \multicolumn{2}{c|}{} & \multicolumn{4}{c|}{ Septiembre } & \multicolumn{3}{c|}{ Diciembre } \\
\cline { 3 - 10 } \multicolumn{2}{c|}{} & $09: 00$ & $09: 30$ & $10: 00$ & $10: 30$ & $09: 00$ & $09: 30$ & $10: 00$ & $10: 30$ \\
\hline 1 & Ev & 720,01 & 1560,45 & 850,21 & 360,09 & 530,01 & 260,01 & 406,07 & 300,41 \\
\hline 2 & Ev & 700,54 & 620,23 & 830,05 & 810,45 & 240,78 & 141,18 & 280,91 & 220,6 \\
\hline 3 & Ev & 472,78 & 612,04 & 576,15 & 513,78 & 240,12 & 442,65 & 610,14 & 540,45 \\
\hline 4 & Ev & 680,12 & 820,12 & 560,09 & 350,45 & 295,09 & 374,78 & 478,95 & 375,48 \\
\hline 5 & Ev & 612,21 & 576,54 & 513,14 & 313,12 & 440,07 & 350,78 & 564,22 & 320,07 \\
\hline 6 & Ev & 490,69 & 940,47 & 760,15 & 547,08 & 570,45 & 260,38 & 550,46 & 425,64 \\
\hline 7 & Ev & 650,12 & 950,45 & 840,08 & 800,12 & 580,46 & 280,14 & 460,09 & 570,47 \\
\hline
\end{tabular}

Para saber si esta variación en GSV se debe al efecto planteado en la hipótesis o a factores fotométricos se realizaron los siguientes análisis:

\subsection{Iluminancia vertical}

La tabla 2 muestra los valores de iluminancia vertical media (Ev) desde las 9 a 10:30am (rango horario en que se registraron manchas en el campo de visión de los ocupantes). Los valores de temperatura y humedad fueron en septiembre $\mathrm{T}^{\circ}$ $(\mathrm{m}=21, \mathrm{Ds}=2)$, humedad $(40 \%)$ y en diciembre $\mathrm{T}^{\mathrm{o}}(\mathrm{m}=30,02$, Ds=1.5) y humedad (37\%).

Los valores de Ev fueron inferiores en diciembre $(\mathrm{m}=394,39$, $\mathrm{Ds}=144,64)$ que en septiembre $(m=683$, Ds=257.16). Las pruebas Wilcoxon para muestras relacionadas muestran que hubo diferencias significativas entre las dos situaciones $(\mathrm{p}<\mathrm{o}, 001)$. Los participantes permitieron en sus oficinas valores de Ev menores a 150olx coincidiendo con los valores recomendados por la comunidad científica $(5,29)$. En septiembre, la correlación entre GSV y Ev fue de rho=0,51; también se calculó un $\mathrm{R}^{2}=0,30$ con un valor de $\mathrm{p}=0,003$. Lo que indica que la Ev contribuyó a la sensación de deslumbramiento. En diciembre la correlación entre GSV y Ev fue de rho=0,33 con un $\mathrm{R}^{2}=0,19$ y valor de $\mathrm{p}=0,03$. Indicando que la contribución de Ev fue un poco menor para este mes.

\subsection{Uniformidad de iluminancias}

Respecto a los valores de uniformidad, todas las oficinas tuvieron valores menores a 0,5 ; es decir que se definieron como no uniformes, este resultado es esperable ya que todos los puestos de trabajo tuvieron manchas de luz directa sobre el plano horizontal.

\subsection{Deslumbramiento (contraste de luminancias y modelo DGP)}

Respecto al deslumbramiento calculado, a través del modelo DGP, podemos decir que todas las oficinas durante su horario de ocupación tuvieron un deslumbramiento menor a o,30 lo que equivale a un valor de "imperceptible", esto es debido a que las Ev no superaron los 150olx en ninguna condición y el DGP basa sus predicciones en esta variable. Como las Ev fueron relativamente bajas, el cálculo de deslumbramiento también lo fue. Por otro lado, la correlación entre DGP y la percepción de deslumbramiento GSV en septiembre fue rho $=0,46$ mientras que en diciembre fue rho=0,26; observándose un valor de correlación más bajo para este último mes, debido a que las Ev registradas en esta condición fueron más constantes.
Respecto al contraste de luminancias, para el mes de septiembre, el contraste de luminancias entre tarea y fuente del campo de visión cercano fue superior a los niveles recomendados (1:10) para todos los participantes en al menos un periodo de la jornada. Estuvieron en rangos de luminancias aceptables 13 de las 28 situaciones lumínicas. Lo mismo para el contraste de luminancias entre tarea y fuente del campo de visión lejano. Para el mes de diciembre, el contraste de luminancias entre tarea y fuente del campo de visión cercano fueron similares al mes de septiembre aunque con un pequeño incremento de las situaciones lumínicas en rango aceptable (17 de las 28 situaciones lumínicas). Este comportamiento lumínico se puede observar con mayor claridad a través de la tabla 3.

Tabla 3. Porcentaje de luminancias en rango aceptable por período estacional.

\begin{tabular}{|l|c|c|c|c|}
\cline { 2 - 5 } \multicolumn{1}{c|}{} & $09: 00$ & $09: 30$ & $10: 00$ & $10: 30$ \\
\hline Septiembre & $28 \%$ & $85 \%$ & $28 \%$ & $28 \%$ \\
\hline Diciembre & $42 \%$ & $100 \%$ & $14 \%$ & $42 \%$ \\
\hline
\end{tabular}

Como se puede observar en la tabla 3 el porcentaje de luminancias en rango aceptable alcanza su mayor nivel a las 09:30 (85\% para septiembre y 100\% para diciembre) a partir de esta hora disminuyen las relaciones de luminancia en rango aceptable. Reforzando la hipótesis de habituación al deslumbramiento, ya que a pesar de incrementarse los niveles de contraste de luminancias, la sensación de deslumbramiento disminuyó.

Las pruebas estadísticas que se presentan a continuación refuerzan esta última afirmación: la correlación entre el contraste de luminancias (Lt/Ls_cvc) y GSV en septiembre fue rho $=0,25$, con un $\mathrm{R}^{2}=0,06$ y valor de $\mathrm{p}=0,18$. Mientras que en diciembre fue $\mathrm{rho}=0,29$, con un $\mathrm{R}^{2}=0,107$ y valor de $\mathrm{p}$ $=0,14$. La correlación entre el contraste de luminancias (Lt/ Ls_cvl) y GSV en septiembre fue rho $=0,18$, con un $\mathrm{R}^{2}=0,001$ $\mathrm{y}$ valor de $\mathrm{p}=0,98$; mientras que en diciembre fue $\mathrm{rho}=0.03$, con un $\mathrm{R}^{2}=0,01 \mathrm{y}$ valor de $\mathrm{p}=0,85$. A partir de este análisis podemos decir que el contraste de luminancias tiene una correlación baja con GSV. Los valores bajos de $\mathrm{R}^{2}$ con valores de $\mathrm{p}$ no significativos indican que las relaciones de luminancia no contribuyeron a la sensación de deslumbramiento observada y que la disminución en la sensación de deslumbramiento puede ser atribuida al factor temporal.

\subsection{Registro del uso de persianas}

A partir del registro del uso de las persianas cada 30 minutos se observó que en ambos meses el uso fue bajo (tabla 4), lo que implica que las persianas estuvieron mayormente fijas: 
En la condición de septiembre las persianas estuvieron en $45^{\circ}$ es decir semi-abiertas permitiendo un mayor ingreso de la radiación directa. Por el contrario, en la condición de diciembre, las persianas estuvieron mayormente semi-cerradas en $15^{\circ}$, registrándose sólo una oficina con las persianas en $\mathrm{O}^{\mathrm{O}}$ y bloqueo total del sol. Es decir que en todas las oficinas durante diciembre hubo una mayor restricción del ingreso de sol, evitando las manchas de sol en el área de visión. Este comportamiento de los oficinistas se ve reflejado en los valores lumínicos registrados (iluminancias, mapeos de luminancias y relaciones de luminancias).

Tabla 4. Porcentaje que oficinas con parasoles en $0^{\circ}, 15^{\circ}, 45^{\circ}$ y $90^{\circ}$.

\begin{tabular}{|c|c|c|c|c|c|}
\cline { 2 - 6 } \multicolumn{1}{c|}{} & Ángulo & $09: 00$ & $09: 30$ & $10: 00$ & $10: 30$ \\
\hline \multirow{3}{*}{ septiembre } & $45^{\circ}$ & $28 \%$ & $100 \%$ & $100 \%$ & $100 \%$ \\
\cline { 2 - 6 } & $90^{\circ}$ & $72 \%$ & $0 \%$ & $0 \%$ & $0 \%$ \\
\hline \multirow{3}{*}{ diciembre } & $0^{\circ}$ & $16 \%$ & $0 \%$ & $14,50 \%$ & $29,00 \%$ \\
\cline { 2 - 6 } & $15^{\circ}$ & $42 \%$ & $75.5 \%$ & $71 \%$ & $71 \%$ \\
\cline { 2 - 6 } & $45^{\circ}$ & $42 \%$ & $14,50 \%$ & $14,50 \%$ & $0,00 \%$ \\
\hline
\end{tabular}

\subsection{Discusión de los datos obtenidos}

Es importante destacar que si bien se preveía que las personas toleraran más las manchas de sol en diciembre por estar menos tiempo sobre el campo de visión (datos de simulación), los resultados (acciones de las personas) no coinciden con los resultados esperados. En diciembre se bloqueó el ingreso de la mancha a primera hora de la mañana restringiendo el ingreso de sol el resto de la jornada, por motivos térmicos. Por otro lado, en septiembre el uso de los parasoles fue menor, permitiendo el ingreso de las manchas de sol. Estas manchas fueron más toleradas a pesar de estar más tiempo en el campo de visión. Hablamos de una mayor tolerancia al deslumbramiento en septiembre, porque a pesar de haberse registrado periodos de la jornada con deslumbramiento "perturbador" los participantes teniendo la posibilidad de modificar su entorno, no lo hicieron.

Respecto a los valores de $\mathrm{R}^{2}$ de las posibles variables explicativas, podemos decir que, por un lado, los contrastes de luminancias (CL) fueron muy bajos y no significativos. Por otro lado, si bien los $\mathrm{R}^{2}$ de la iluminancia vertical fueron bajos ( $\mathrm{r} 2=0,29, \mathrm{p}=0,003$ ), fue la única medida fotométrica con un valor p significativo. Es decir, que contribuyó a la sensación de deslumbramiento, por lo que se la analiza en profundidad en la sección siguiente. Como la contribución de la Ev fue baja podemos decir que la disminución de la sensación de deslumbramiento registrada a lo largo de la jornada en el mes de septiembre indica un posible efecto de habituación al deslumbramiento, relacionada con la hipótesis de Macfarlane.

\subsection{Variación temporal de la sensación de deslumbramiento}

Las figura 6 y 7 muestra los valores de GSV para cada participante en función de Ev.

La figura 6 muestra la disminución de la sensación de deslumbramiento (GSV) a lo largo de la jornada para el mes de septiembre. Como se puede observar las iluminancias verticales se mantuvieron en una franja de entre 400 a 9oolx excepto para el participante 3 con un pico de iluminancia vertical de 150olx.
La figura 7 muestra la sensación de deslumbramiento a lo largo de la jornada para el mes de diciembre. GSV fue alternando entre notable e imperceptible.

Para poder observar mejor el efecto temporal de la sensación de deslumbramiento re-codificamos los dos primeros valores de GSV (imperceptible-notable) como 'Sin sensación de deslumbramiento molesto' y los dos últimos valores (perturbador-intolerable) como 'Con sensación de deslumbramiento molesto'. La tabla 5 y 6 muestran el porcentaje de oficinistas registrados "Sin sensación de deslumbramiento molesto" y "Con sensación de deslumbramiento molesto" cada media hora. En estas tablas se puede ver con mayor claridad el efecto que se observa en la figura 6 y 7.

Se registró un $42 \%$ de oficinistas con deslumbramiento entre las 09:00 y 9:30, y a partir de las 9:30 el valor de personas deslumbradas se reduce a $0 \%$. Este efecto puede

\section{Septiembre}

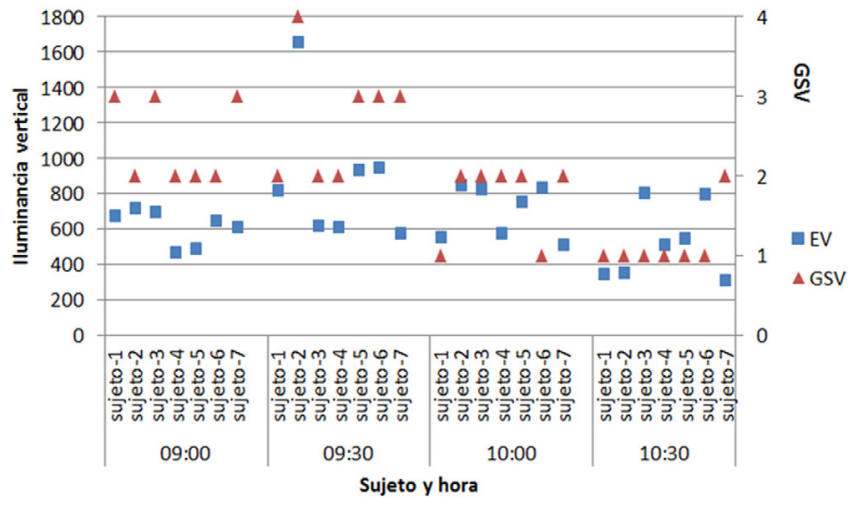

Figura 6. Sensación de deslumbramiento en función de la iluminancia vertical al ojo para cada sujeto. Eje (x) respuesta de los sujetos cada media hora, eje (y izquierda) iluminancia vertical al ojo, eje (y derecha) sensación de deslumbramiento (GSV).

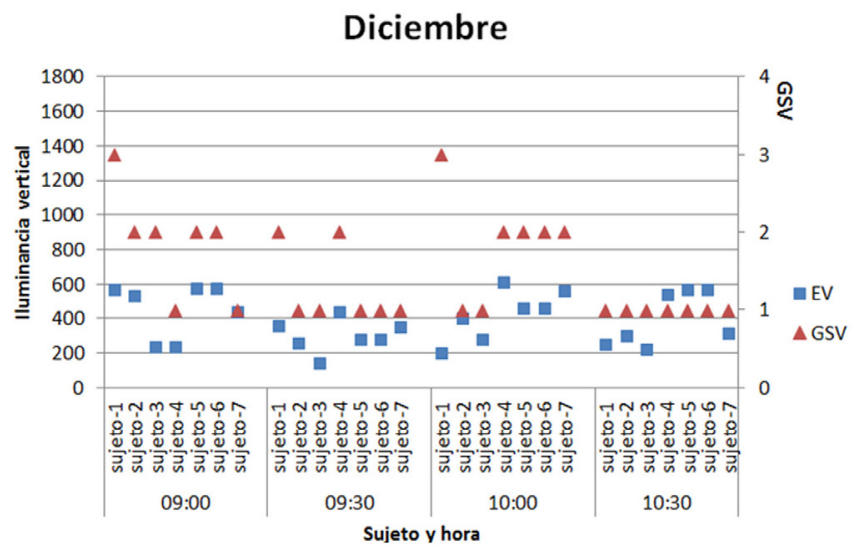

Figura 7. Sensación de deslumbramiento en función de la iluminancia vertical al ojo para cada sujeto. Eje (x) respuesta de los sujetos cada media hora, eje (y izquierda) iluminancia vertical al ojo, eje (y derecha) sensación de deslumbramiento (GSV).

Tabla 5. Porcentaje de personas con y sin deslumbramiento por periodo horario para el mes de septiembre.

\begin{tabular}{|l|c|c|c|c|c|}
\hline Septiembre & $09: 00$ & $09: 30$ & $10: 00$ & $10: 30$ & $11: 00$ \\
\hline Con-deslumbr. & $42,85 \%$ & $42,85 \%$ & O\% & $0 \%$ & $0 \%$ \\
\hline Sin-deslumbr. & $57,14 \%$ & $57,14 \%$ & $100 \%$ & $100 \%$ & $100 \%$ \\
\hline
\end{tabular}


ser atribuido en pequeña medida a la principal variable explicativa encontrada $\mathrm{Ev}(\mathrm{R} 2=0,30)$, parte de la varianza no explicada por esta variable puede ser atribuida a un factor temporal.

Tabla 6. Porcentaje de personas con y sin deslumbramiento por periodo horario para el mes de diciembre.

\begin{tabular}{|l|c|c|c|c|c|}
\hline Diciembre & 09:00 & $09: 30$ & $10: 00$ & $10: 30$ & $11: 00$ \\
\hline Con-deslumbr. & $14,28 \%$ & $0 \%$ & $14,28 \%$ & $0 \%$ & $0 \%$ \\
\hline Sin.deslumb. & $85,71 \%$ & $100 \%$ & $85,71 \%$ & $100 \%$ & $100 \%$ \\
\hline
\end{tabular}

\subsection{Limitaciones de este trabajo}

Es importante mencionar ciertas variables que podrían influir en la percepción de deslumbramiento y que no fueron registradas ni evaluadas en este trabajo. Estas fueron: La temperatura de color correlacionada (30), las diferencias individuales (11) y el comportamiento activo de los ocupantes (31).

\section{CONCLUSIONES}

Los estudios observacionales proporcionan información valiosa, ya que le permiten al investigador detectar el tipo y la magnitud de variables que influyen en un determinado fenómeno, en este caso en el deslumbramiento. A pesar de haber trabajado con una muestra pequeña, la estadística paramétrica nos permitió realizar comprobaciones de validez y fiabilidad, como así también permitirá la posibilidad de replicar este estudio.

De la evaluación objetiva de deslumbramiento podemos decir que, respecto a los niveles de iluminancia vertical, estos estuvieron dentro de un rango aceptable según recomendaciones recientes de la comunidad científica $(<1500 l x)$. Análisis que no coincidió con el análisis de luminancias, donde encontramos que: El contraste de luminancias fue superior a los valores recomendados (1:10:20) en al menos un período de la jor- nada. Cabe destacar que estas situaciones fueron permitidas y/o generadas por los ocupantes.

Respecto a la evaluación subjetiva de deslumbramiento podemos decir que, por un lado, en diciembre con un mayor control del ingreso de radiación directa y niveles lumínicos más bajos, se observó una sensación de deslumbramiento menor (imperceptible-notable). Por otro lado, en septiembre con un menor control del ingreso de radiación directa y mayores niveles lumínicos, se observó una mayor sensación de deslumbramiento al inicio de la jornada (notableperturbador) que fue disminuyendo en el transcurso de la misma (notable-imperceptible). Esta disminución de la sensación de deslumbramiento a lo largo de la jornada en el mes de septiembre indica un posible efecto de habituación al deslumbramiento, relacionada con la hipótesis de Macfarlane (32), que sostiene que una fuente de deslumbramiento prolongada en el tiempo produce la reducción de la respuesta del organismo (Macfarlane 1970).

Sin embargo, si comparamos los dos periodos estacionales (septiembre y diciembre) cabe destacar que jugó un papel más importante en la sensación de deslumbramiento la cantidad de luz que el tiempo. Es decir que, en diciembre se bloqueó el ingreso de radiación directa para tener niveles lumínicos más bajos, para reducir el deslumbramiento y el sobrecalentamiento del espacio. Sin embargo, y como se mencionó anteriormente, en septiembre las personas toleraron el deslumbramiento al inicio de la jornada, posiblemente por el corto periodo de tiempo que este estuvo en el campo de visión.

Los datos sugieren que las personas tendrían una mayor tolerancia al deslumbramiento por el efecto transitorio de la fuente en ciertas condiciones, como por ejemplo, cuando el deslumbramiento no es extremo. Si se logra aislar este factor de otras variables como la TCC, las diferencias individuales, etc., podríamos incorporar un factor temporal a la sensación de deslumbramiento. Presentándose a este factor temporal como un estresor positivo.

\section{REFERENCIAS}

(1) Pattini, A. (2007). Diseño de artefactos de iluminación natural. La utilización de la energía solar para iluminar espacios interiores (Informe final de proyecto). Universidad Nacional de Cuyo, Secretaría de Ciencia, Técnica y Posgrado.

(2) Boyce, P. (2014). Human factors in lighting. 3rd ed. Boca Ratón, Florida: Taylor y Francis Crc Press.

(3) Villalba, A.M., Pattini, A.E., Córica, M.L. (2012). Análisis de las características morfológicas de las envolventes edilicias y del entorno urbano desde la perspectiva de la iluminación natural. Ambiente Construído, 12(4): 159-75. https://doi. org/10.1590/S1678-86212012000400011

(4) DiLaura, D.L., Houser, K.W., Mistrick, R.G., Steffy, G.R. (2010). The lighting handbook: Reference and application. Illuminating Engineering Society of North America. New York (NY).

(5) Wienold, J. (2009). Daylight Glare in Offices (PhD thesis). Universität Karlsruhe.

(6) Wienold, J., Christoffersen, J. (2006). Evaluation methods and development of a new glare prediction model for daylight environments with the use of CCD cameras. Energy and Buildings, 38(7): 743-57. https://doi.org/10.1016/j. enbuild.2006.03.017

(7) Pierson, C., Wienold, J., Bodart, M. (2017). Discomfort glare perception in daylighting: influencing factors. Energy Procedia, 122: 331-336. https://doi.org/10.1016/j.egypro.2017.07.332

(8) Pulpitlova, J., Detkova, P. (1993). Impact of the cultural and social background on the visual perception in living and working perception. In Proceedings of the international symposium 'Design of amenity.

(9) Tuaycharoen, N., Tregenza, P.R. (2007). View and discomfort glare from windows. Lighting Research \& Technology, 39(2): 185-200. https://doi.org/10.1177/1365782807077193

(10) Wang, N., Boubekri, M. (2014). Design recommendations based on cognitive, mood and preference assessments in a sunlit workspace. Lighting Research \& Technology, 43(1): 55-72. https://doi.org/10.1177/1477153510370807 
(11) Rodriguez, R.G., Pattini, A. (2012). Tolerance of discomfort glare from a large area source for work on a visual display. Lighting Research \& Technology, 46(2): 157-170. https://doi.org/10.1177/1477153512470386

(12) Yamin Garretón, J., Rodriguez, R.G., Ruiz, A., Pattini, A.E. (2015). Degree of eye opening: A new discomfort glare indicator. Building and Environment, 88: 142-150. https://doi.org/10.1016/j.buildenv.2014.11.010

(13) Yamin, J.A., Rodriguez, R., Pattini, A. (2015). Effects of perceived indoor temperature on daylight glare perception. Building Research \& Information, 44(8): 907-919. https://doi.org/10.1080/09613218.2016.1103116

(14) Kent, M.G., Altomonte, S., Wilson, R., Tregenza, P.R. (2017). Temporal effects on glare response from daylight. Building and Environment, 113: 49-64. https://doi.org/10.1016/j.buildenv.2016.09.002

(15) Clear, R.D. (2013). Discomfort glare: What do we actually know?. Lighting Research \& Technology, 45(2): $141-158$. https://doi.org/10.1177/1477153512444527

(16) Macfarlane, W. (1974). Habituation. Adelaide, Australia: University of Adelaide.

(17) Kent, M.G., Altomonte, S., Tregenza, P.R., Wilson, R. (2014). Discomfort glare and time of day. Lighting Research \& Technology, 47(6): 641-657. https://doi.org/10.1177/1477153514547291

(18) Osterhaus, W.K.E. (1996). Discomfort glare from large area glare sources at computer workstations. In Proceedings for the 1996 International Daylight Workshop, Building with Daylight: Energy-Efficient Design (p. 103-110). Perth, Australia: University of Western Australia.

(19) Osterhaus, W.K.E., Bailey, I.L. (1992). Large area glare sources and their effect on visual discomfort and visual performance at computer workstations. In Industry Applications Society Annual Meeting, Conference Record of the 1992 IEEE, vol. II, pp. 1825-1829. https://doi.org/10.1109/IAS.1992.244537

(20) Yamin, J.A., Colombo, E.M., Pattini, A.E. (2018). A global evaluation of discomfort glare metrics in real office spaces with presence of direct sunlight. Energy and Buildings, 166: 145-153. https://doi.org/10.1016/j.enbuild.2018.01.024

(21) Hopkinson, R.G. (1957). Evaluation of glare. Illuminating Engineering, 52(6): 305-316.

(22) Suk, J.Y., Schiler, M., Kensek, K. (2016). Absolute glare factor and relative glare factor based metric: Predicting and quantifying levels of daylight glare in office space. Energy and Building, 130: 8-19. https://doi.org/10.1016/j.enbuild.2016.08.021

(23) Inanici, M.N. (2006). Evaluation of high dynamic range photography as a luminance data acquisition system. Lighting Research \& Technology, 38(2): 123-34. https://doi.org/10.1191/1365782806li1640a

(24) Wienold, J. (2015). Evalglare (version v1.22), pp. 1-4.

(25) Kumaragurubaran, V., Inanici, M. (2013). Hdrscope: high dynamic range image processing toolkit for lighting simulations and analysis. In Proceedings of the BS2013: 13th Conference of International Building Performance Simulation Association, pp. 3400-3407.

(26) Rea, M.S. (2000). The IESNA Lighting Handbook: reference \& application. Illuminating Engineering Society of North America.

(27) Iwata, T., Tokura, M. (1998). Examination of the limitations of predicted glare sensation vote (PGSV) as a glare index for a large source Towards a comprehensive development of discomfort glare evaluation. Lighting Research \& Technology, 30(2): 81-88. https://doi.org/10.1177/096032719803000205

(28) Gómez-Gómez, M., Danglot-Banck, C., Vega-Franco, L. (2003). Sinopsis de pruebas estadísticas no paramétricas. Cuándo usarlas. Revista Mexicana de Pediatría, 70(2): 91-99.

(29) Konstantzos, I., Tzempelikos, A., Chan, Y-C. (2015). Experimental and simulation analysis of daylight glare probability in offices with dynamic window shades. Building and Environment, 87: 244-54. https://doi.org/10.1016/j.buildenv.2015.02.007

(30) Kobayashi, H., Sato, M. (1992). Physiological responses to illuminance and color temperature of lighting. The Annals of physiological anthropology, 11(1): 45-49. https://doi.org/10.2114/ahs1983.11.45

(31) Reinhart, C.F. (2004). Lightswitch-2002: a model for manual and automated control of electric lighting and blinds. Solar energy, 77(1): 15-28. https://doi.org/10.1016/j.solener.2004.04.003

(32) Macfarlane, W. (1970). Habituation. In Progress in Biometeorology. Division A Progress in Human Biometeorology, 1 Pt 1B: 462-467. 